\title{
XVII. Notice of an instrument for ascertaining various properties of terrestrial magnetism, and affording a permanent standard measure of its intensity in every latitude
}

\section{Robert Were Fox}

To cite this article: Robert Were Fox (1834) XVII. Notice of an instrument for ascertaining various properties of terrestrial magnetism, and affording a permanent standard measure of its intensity in every latitude, Philosophical Magazine Series 3, 4:20, 81-88, DOI:

10.1080/14786443408648267

To link to this article: http://dx.doi.org/10.1080/14786443408648267

曲 Published online: 01 Jun 2009.

Submit your article to this journal $₫$

Џll Article views: 7

Q View related articles ¿

47 Citing articles: 1 View citing articles 진 
TH F:

\title{
LONDON AND EDINBURGH
}

\section{PHILOSOPHICAL MAGAZINE}

\author{
AND \\ JOURNAL OF SCIENCE. \\ [THIRD SERIES.]
}

$F E B R U A R Y 1834$.

XVII. Notice of an Instrument for ascertaining various Properties of Terrestrial Magnetism, and offording a permanent Standard Measure of its Intensity in every Latitude. By Robert Were Fox*.

TNTERESTING as is the subject of terrestrial magnetism, our acquaintance with many of its phænomena is very limited, owing principally to the imperfection of our instruments, and the time and minute attention which the best of them require, to enable us to obtain results which can be considered even as approximations to accuracy. Impressed with these defects, I have directed some attention to the subject, and have succeeded in constructing an instrument, which I think will be found highly useful in the prosecution of magnetical researches in any part of the world.

It consists of a dipping-needle with a transverse axis, and pivots working in jewel holes, like those used for the balance of a chronometer: the needle is contained in a vertical box of the usual form, having a concentric brass plate inserted in the back, which admits of its being turned round on its axis independently of the box. The jewels are set precisely in this axis, one in the concentric plate itself, and the other in an arm connecting the axis with the plate. This contrivance admits of the needle's taking any direction without interruption by the arm, which is moveable by pins affixed to the back of the plate. In the construction of this instrument, every precaution usually taken for the true adjustment of a dipping-needle should be observed, the friction of the pivots

* Communicated by the Author.

Third Series. Vol. 4. No. 20. Feb. 1834. 
being overcome by a slight vibration of the concentric plate. This vibration may be produced by gently tapping the plate at the back, or the latter may be indented, so that the passing of a brass rod once or twice rapidly over its surface may, by the friction produced, effect the desired object. After a little practice I have found that this plan causes the needle to take its natural bearing in the most satisfactory manner, and at least to equal in delicacy any other method of mounting it. The perpendicular support of the box rests on a horizontal plane or base, and can be turned round on its axis in any direction, and the needle be made to face any point of the compass without altering the base of the instrument, which is of course furnished with levels and adjusting screws in the usual manner. Besides the graduated circle on the face of the box, I use another, coinciding with it, which is placed immediately within the glass to direct the line of sight, and to enable the observer to ascertain with great minuteness the place of the needle. For example, if the outer circle be twelve times more distant from the indicating mark on the needle than the latter is from the inner circle, it is evident that any visual lines crossing at the mark, will be separated from each other at the circles in the ratio of the relative distances of the latter from the mark; so that a degree on the outer circle will in this way represent one twelfth, or five minutes of a degree on the inner one. This mode of division need only be limited by our means of observation.

'The plane of the magnetic meridian may be easily found without a horizontal compass, by moving the face of the instrument round till the needle becomes vertical (after vibration), which will be its position when it is exactly opposite the magnetic north or south, so that the needle will be in the plane required when turned $90^{\circ}$ from that point. If the base of the instrument be furnished with a graduated scale round the circular edge of the support or axis on which the box revolves, and the axis itself with a vernier, or vice vers $\hat{a}$, it will enable us to ascertain the variation of the magnetic from the true meridian with great precision. If from defects in the construction of the needle (to which all are liable,) its magnetic axis and centre of gravity should be so untrue as to prevent its being vertical when placed at right angles to the plane of the magnetic meridian, the error will be shown, and its extent detected, by repeating the observations with the face of the instrument turned towards the opposite quarter; for in this case, if the azimuth distance, when the needle again becomes vertical, should exceed or fall short of $180^{\circ}$, the difference will show the correction that must be applied. This property of 
self-correction, which may be relied upon for the certainty of its results, gives this mode of taking the magnetic variation a decided advantage over the common method with the azimuth compass.

The true meridian may, of course, be found in the same mamner as in the azimuth compass; but as the vertical box has a graduated circle at its back agreeing with those within it, and a fixed pivot, the axis of which is in the common centre, (for reasons immediately to be stated,) I intend to adapt to it a tube on the principle of those used in theodolites, having a broad flanch at each end, the solar rays being transmitted through slits at right angles to each other in the flanch at the object end, and received by the other at the opposite end, which is faced with ivory to render the lines of Jight more distinct ; and I may fix to one or more of these slits the segment of a glass cylinder, as adopted by Capt. Kater in his azimuth compass, for the purpose of increasing this effect. This tube, being fitted to a bar which turns on the central pivot, and furnished with verniers at each end, suited to the graduated circle, will enable the observer to find the sun's meridian and altitude at the same time. The true adjustment of the tube may be ensured, or any needful corrections ascertained, by taking the observations with the face of the box turned in opposite directions. The inside of the tube may be provided with cross wires and glasses, so as to render the instrument a good theodolite, if desired.

But the most valuable property of the instrument, because the most wanted, is the facility with which it will indicate the intensity of the earth's magnetism in every latitude. To accomplish this object, steel magnets are employed to deflect the needle from its natural dip, the greater or less intensity existing at the place of observation being determined by the extent of the deflection. I have employed two magnets, each three inches in length (or about one half that of the needle), and after having been exposed to the heat of $150^{\circ}$ at least, I have inclosed them in two brass tubes. These are made to slide in a larger tube, six inches in length, on the principle of the spy-glass, one being fixed at each end, so that the magnetic poles alternate with each other. The large tube is perforated through the middle, and exactly fits the central pivot at the back of the box, where it may be confined in its place by means of a nut and screw at the end of the pivot, or taken off at pleasure. This arrangement admits of the tube or deflector being turned round in a vertical plane parallel to that in which the dipping-needle moves, and of its being firmly fixed in any required position within that plane. 


\section{Mr. R. W. Fox's Notice of an Instrument for ascertaining}

The deflector is furnished with verniers at its extremities. To ascertain the magnetic intensity, let the deflector (with the tubes closed, for example,) have its poles turned round so as to coincide as nearly as possible with the dip of the needle at the place of observation; the latter will be deflected either to the right or left (the amount of this deflection should not, I think, be much less than $60^{\circ}$ or $70^{\circ}$ ); produce vibration and then note the place of the needle, observing it at both ends. Draw out one of the small tubes for a moment, till the needle swings back beyond the line of the dip; then quickly return the tube to its former position, and the needle will be deflected in the opposite direction: half the sum of these deflections will represent the intensity of the terrestrial magnetism at the place of observation. This should be repeated with the face of the box turned the opposite way, the deflector being made to coincide with the dip as before. The small tubes and the deflector being turned round $180^{\circ}$, the observations should be again repeated in like manner, and will tend to correct the previous results. In this case it is evident that the poles of the deflecting magnets are opposed to the direction of the terrestrial magnetism, whereas before they coincided with it, and its influence on the force of the magnets becomes hereby compensated. The deflectors may be employed in attracting instead of repelling the needle, by being placed at right angles or at any other given angle to the dip; but there are many reasons, I think, for preferring the former. The deflector may also be used with great advantage in correcting the observed dip, inasmuch as the force of the earth's magnetism on the needle increases in the ratio of the sine of the angle of deflection. This it may effect in different ways; but a small deflection appears to me to give the most satisfactory and decisive results; and the force of the deflector may in this case be modified by drawing out both tubes, more or less, and adjusting it at any given angle on each side of the dip. The effect of temperature on the deflector and dipping-needle may be easily ascertained by covering the instrument with a double metallic case, containing water at any required temperature, an opening being left in it for the purpose of observation, which should be covered with glass. It will, however, be sufficient for the maker, to ascertain what corrections should be made, as it respects each instrument, on this account. I have employed such an apparatus, and found that a needle repelled by a deflector at an angle of $61^{\circ} 30^{\prime}$ from it when the temperature was $52^{\circ}$, stood at $61^{\circ} 15^{\prime}$ when the temperature was raised to $95^{\circ}$, and returned to $6 l^{\circ} 30^{\prime}$ on cooling; so that the amount of 
deflection was reduced only $\frac{1}{4}^{\frac{1}{4}} 5^{\text {th }}$ part by an increase of temperature equal to $43^{\circ}$. It may here be remarked, that the effect of temperature on the reciprocal influence of the deflector and needle, is in part compensated by a corresponding change of the effect of the earth's magnetism on the latter. I am about to apply to my instrument a simple contrivance for limiting the distance to which the magnetic tubes may be drawn out or pushed in, according to the temperature existing at the time; but its influence is so inconsiderable, and corrections can be so easily applied to the results, that it does not seem necessary to describe it. It is always desirable to note the temperature at the time of observation, and a small thermometer is affixed to the face of the box for this purpose.

I am aware that a vibrating needle or any moderately light body in constant motion, is liable to be much affected by temperature in consequence of the mechanical* action of the air, in which currents are produced by the application of warmth; thus experiments on the oscillations of the needle, and even of the pendulum in a small degree, are liable to more or less uncertainty on this account, the anomalous and fluctuating results obtained from the former being often very remarkable.

When the apparatus is not in use, the ends of the needle are received into sockets attached to brass springs fixed to the side of the box, and which may be pushed forward by screws passing through it. The deflectors at the same time should be turned round so as to be parallel to the needle, but with its poles reversed. With this precaution, I have reason to believe that the magnetic forces will, within a moderate time, become sufficiently permanent for making satisfactory comparisons relative to the magnetic intensity in different parts of the world: at any rate, if there should be any diminution of these forces after the instrument has been completed twelve months, it will, (protected as the magnets are, I believe, be very inconsiderable, and its ratio continually diminishingt.

- This source of error having been referred to by others, I think it proper to say that I believe I was the first person to announce it, early in 1832. There is also a paper of mine on the subject since inserted in the Lond. and Edinb. Phil. Mag. vol. i. p. 310.

+ In corroboration of this opinion, I have found that a horizontal magnetic needle, or rather bar, coated with sealing-wax, and suspended by unspun silk in a box, in my undergronnd cellar, vearly three years ago, has suffered no sensible change of intensity in the last twelve months; or at least, the time in which it performs a given number of vibrations does not differ so much as one second in $1434^{\prime \prime}$. The temperature there fluctuates very little, and it was at $52^{\circ}$ when these comparative results were obtained. 


\section{$86 \mathrm{Mr}$. R. W. Fox's Notice of an Instrument for ascerlaining}

The instrument in question will, however, admit of the application of an unequivocal test of any change in its magnetic force.

If the dipping-needle be made to assume a horizontal position, by the proper adjustment of the deflector, or by weights applied to its southern arms, it will retain very nearly this position, whatever way it may be turned; and it would quite do so, were it not for the action of the terrestrial magnetism on the deflector and needle, which tends to produce some modification of their forces, in a greater or less degree, according to their relative directions: for example, if the north pole of the needle be deflected upwards from its natural dip in this latitude when in the plane of the magnetic meridian, by raising the north pole of the deflector under it (with the deflecting tubes closed,) till the needle is perfectly horizontal and pointing towards the north, both the needle and deflector will have their forces augmented by the action of the terrestrial magnetism; and the latter more so than the former, because it more nearly approaches the direction of the magnetic currents. Turn the face of the instrument round $90^{\circ}$, when the terrestrial magnetism will have no effect in modifying the force of the horizontal needle, though it will still add to that of the deflector; in this case the needle will have a tendency to suffer a slight depression. Let the instrument be turned $90^{\circ}$ further round till it is again in the plane of the magnetic meridian, but with the north end of the needle pointing towards the south; the earth's magnetism will in this case tend to diminish the force of the needle and of the deflector also, if it be at a greater angle from the horizontal line than the complement of the dip, and the depression of the north pole of the needle will probably not be less, and may be more considerable than when the instrument was at right angles to the plane of the magnetic meridian *. This source of error may, however, be compensated by reversing the direction of the deflector, and drawing out the tubes: so that the mean results obtained in both these ways, when the needle is at right angles to the plane

- The modifying effect of the terrestrial magnetism is rendered more apparent by attaching small weights to the north pole of the needle, and raising the deflector till the needle becomes horizontal.

Does it not seem to follow that the results obtained by the vibrations of a magnetic needle are influenced, not only by the attraction of the terrestrial magnetism,'but also by an adventitious force, which it imparts to the needle in a greater or less degree in proportion as their relative directions are more or less coincident with each other? I do not know whether this question has been duly considered, but it appears to me to be of some importance that it should be taken into account in making comparative observations in different latitudes with the horizontal vibrating needle. 
of the magnetic meridian, may be consideled as independent of any modification arising from the earth's magnetism.

Let the north pole of the needle be deflected above the horizontal line, the deflector being fixed at some given angle from it: suspend minute weights from the northern arm at any given point, say, for instance, midway between the centre and extremity of the needle, till it is reduced to the horizontal position. The weights required to effect this will represent the force of the deflection minus the directive influence of the earth's magnetism on the needle so circumstanced. Remove the weights, and raise the north pole of the deflector as many degrees above the horizontal line as it was before below it, when the deflecting force will cooperate with that of the terrestrial magnetism, and the weights required to render the needle horizontal, if applied to the corresponding side on the southern arm, will be more than in the former case at every place north of the magnetic equator. One half the excess or difference in the weights will represent the directive force of the terrestrial magnetism, acting on the needle in question when horizontal, and half the sum of the weights employed on both arms of the needle will indicate the force with which it is deffected under the actual circumstances of the case. The latter will be a constant quantity, (i.e. the mean of the results obtained with the deflector turned in opposite directions and the tubes closed, and afterwards drawn out, as before described,) whilst the forces of the needle and deflector remain unchanged; and if they should have undergone any modification, its extent may be immediately detected by taking one half the weights, however they may be divided, between the two arms. The former quantity or difference of weights on the two arms will be ever varying with the obliquity of the earth's magnetism, and its increased or diminished intensity. The deflecting force, therefore, becomes, I conceive, a standard of comparison for every change which may occur, and the causes of which may hereby be distinguished from each other. Suppose, for instance, the deflecting forces to remain unchanged, and the directive influence of the terrestrial magnetism on the needle to be increased, the southern arm will require an increase, and the northern a diminution of weight; but if the terrestrial force should be diminished, the case will of course be reversed as it respects the weights, their sum remaining the same. If the deflecting forces only, and not the terrestrial magnetism, be increased or diminished, the weights must be increased or diminished on each arm, the proportions of which would be equal, were it not that the directive force 
of the terrestrial magnetism on the needle will undergo some corresponding change, which will bear a constant ratio to the change in the deflecting forces, the extent of which is known.

If the forces of deflection and of the terrestrial magnetism should both change, then, from what has been said above, it appears that the relative amount of each can be estimated.

In the foregoing examples, I have assumed that the defiector and needle suffer equal changes of intensity; and this, $I$ think, they will do (if they change at all), after some time, when they have found their level: but it is well to provide for any contingency; therefore, to detect any alteration which may take place in their relative forces, it is only necessary to substitute the deflecting magnets for the needle, and to allow the latter to deflect the former; and this can be accomplished by inserting the ends of the magnets in two sockets, opposite to each other, and furnished with a transverse axis like that of the needle, so that when balanced on the jewels, the combined magnets may become a dipping-needle, and the amount of its deflection, caused by the needle when properly adjusted in connexion with the verniers at the back, will indicate any change in its force, if such should have occurred.

It may be remarked, as it respects these combined magnets, as well as the dipping-needle itself, that any error in their construction will be always constant when their position is horizontal, and that therefore it will not affect the value of their indications.

It is now more than a year and a half since Watkins and Hill, No. 5, Charing Cross, made my first dipping-needle with a deflector; and I showed it to several scientific individuals in London, and also at Oxford, when I attended the meeting of the British Association in that city. I afterwards had a larger one made in Cornwall, and Watkins and Co. are now constructing another, in which $I$ intend to have some improvements made in the method, above described, of attaching the deflector, \&c., to the back of the instrument.

It is evident that needles, balanced with knife-edges resting on agates, may be used in the manner before mentioned, for obtaining a permanent standard measure of the magnetic intensity; but although this method may be preferred by many to the chronometer one, for local use, I think that it will not be deemed so eligible for locomotive purposes. 